\title{
Planckian Regression Temperature for Least Spectral Error and Least CIELAB Error
}

\author{
Mark S. Drew* and Hamid Reza Vaezi Joze \\ School of Computing Science, Simon Fraser University, \\ Vancouver, B.C., Canada V5A 1 S6 \\ ${ }^{*}$ Corresponding author: $\{$ mark/hrvl $\} @ c s . s f u . c a$
}

\begin{abstract}
Correlated colour temperature (CCT) provides a simple and useful descriptor for a given spectral power distribution as well as an approximation of the full spectrum of the measured illuminant. But typically the CCT is calculated on the basis of distance in the chromaticity plane. Here we suggest that, while familiar, this metric is not the most effective for actually generating a useful spectral approximation. Given the recent interest in whole-spectrum calculations, we consider what optimization would be most sensible for identifying the nearest Planckian in terms of wholespectrum RMS error; in that case we are calculating a variant of the Distribution Temperature (DT), another simple descriptor. This effectively means that instead of one value $T$ we instead describe a spectrum in terms of both $T$ and an intensity $I$. In general, we wish to balance the need for (i) a best mapping of the whole spectrum and (ii) smallest CIELAB error. As a first step we show how to calculate the spectrum analytically in the case when RMS spectral error minimization is the sole goal. Generalizing, we consider an optimization that tries to minimize a balance of RMS and CIELAB error, leading to a family of solutions. Finally we suggest a specific optimization that arguably forms a best tradeoff of these two objectives, which we denote the Planckian Regression Temperature (PRT). Results are shown for some standard test illuminants, and then for a further 102 measured spectra, with results separately reported for fluorescent and non-fluorescent illuminants.
\end{abstract}

(c) 2011 Optical Society of America

OCIS codes: $330.1690,330.1715$

\section{Introduction}

The Correlated Colour Temperature (CCT) is a scalar descriptor characterizing an illuminant spectrum, ostensibly such that the closest Planckian light has the same CCT [1]. Characterizing 
visible light via the CCT is very appealing since it reduces the description down to a single parameter $T$. But of course temperature does not tell the whole story because many disparate spectra can be associated with the same $T$. Nevertheless the CCT has been used in a variety of settings including lighting design, printing, photography, astrophysics and so on. The idea is to correlate a given Spectral Power Distribution (SPD) for an illuminant with the "nearest" blackbody radiator. The assignment of $T$ is usually carried out in chromaticity space [2].

But we may instead be interested in including more information about the shape of the light spectrum, rather than just its closest point on the Planckian chromaticity locus. After all, it would make some sense and would be useful in many situations to simply ask what Planckian spectrum is closest, in a metric such as Least Squares, to a given spectrum. The Distribution Temperature (DT) [2] generates this type of descriptor and includes an intensity term. However the DT is defined in terms of fitting the ratio of a given spectrum to a Planckian distribution to unity (the scalar 1.0); if we fit spectral difference directly, instead of using a ratio, we show here how to generate an analytic solution for both temperature and intensity. Considerable interest has recently been focussed on multispectral processing [3-13] and it makes sense to include at least some aspect of whole-spectrum information into any spectrum descriptor. An example of this type of spectral-based work is [14], where the transfer function used for volume rendering in Graphics is defined in terms of whole spectra, rather than RGB values. Then the panoply of spectral effects such as metamerism and metameric blacks can be brought into play for an interactive visualization of data involving simply changing the spectrum of the light, using a real-time widget. For this example we need to easily characterize spectral data. Moreover in [13] a simple description of spectra could be valuable for generating novel spectral power distributions for use in an optimizer having spectral objectives.

In this paper we show that there is a trade-off between the two goals: (1) characterize the whole spectrum as closely as possible by a Planckian correlate, and (2) find the Planckian closest in perceptual colour space. Moreover, we show that there is a reasonable solution for a single best $T$ that balances these two goals. Each goal is first solved individually, forming natural ending-points for any solution. Then an optimization is introduced that performs a best tradeoff (with a reference implementation supplied online). We call this best $T$ value the Planckian Regression Temperature (PRT).

\section{Chromaticity-Based Methods}

Planck's Law is stated in Physics as [1]

$$
P(\lambda)=k_{1} \lambda^{-5}\left[\exp \left(\frac{k_{2}}{T \lambda}\right)-1\right]^{-1}
$$

with constants $k_{1}$ and $k_{2}$, where temperature $T$ characterizes the lighting colour. Specifically, $k_{1}=3.74183 \times 10^{16} ; k_{2}=1.4388 \times 10^{-2}$ (note that for these units, $\lambda$ is in meters). 
The correlated colour temperature (CCT) is defined by the CIE as the temperature $T$ of the Planckian blackbody whose perceived colour is closest to that of a given spectrum at the same brightness and under specified viewing conditions [15]. Early development included Judd's work on sensitivity to change in colour temperature [16], and subsequently McCamy [17] proposed a cubic equation in terms of $(x, y)$-chromaticity for specifying CCT, as follows:

$$
\begin{aligned}
& n=\left(x-x_{e}\right) /\left(y-y_{e}\right), \quad x_{e}=0.3320, y_{e}=0.1858 \\
& T(x, y)=-449 n^{3}+3525 n^{2}-6823.3 n+5520.33
\end{aligned}
$$

leading to absolute errors for $T$ of less than $2 \mathrm{~K}$ in the range $T=2856 \mathrm{~K}$ for Illuminant $\mathrm{A}$ to $T=6504 \mathrm{~K}$ for D65, relative to the best chromaticity-based $T$.

This temperature range was extended to higher $T$ in [18] by generalizing the above formulation to the following form:

$$
\begin{aligned}
& n=\left(x-x_{e}\right) /\left(y-y_{e}\right), \quad x_{e}=0.3366, y_{e}=0.1735 \\
& T(x, y)=A_{0}+A_{1} \exp \left(-n / t_{1}\right)+A_{2} \exp \left(-n / t_{2}\right)+A_{3} \exp \left(-n / t_{3}\right)
\end{aligned}
$$

with constants given in [18] (here we use the constants for the range $T=3,000-50,000 \mathrm{~K}$ ). Several other suggestions for a best CCT have been posited [19].

As an example, consider the fluorescent illuminant $E(\lambda)$ shown in blue in Fig. 1(a) — "CRT white ( $\simeq 9300 \mathrm{~K}$, white balance artificially set off from the Planckian locus)" [19]. Using the $1931 \mathrm{CIE} 2^{\circ}$ standard observer colour-matching functions [1] (5nm intervals, 380nm-780nm), applying the method of Hernández-Andrés, Lee, and Romero [18] and using the estimate (3) for temperature we find a CCT of 9354K, considerably different than those reported in [19] using other suggested methods. (Note that the result using the McCamy equation (2) is 9274K, which is also quite different from [19].) To go from a chromaticity-based calculation to an absolute one, we also need to go from the Planckian spectral shape to a curve matching the input by multiplying by an intensity $I$. Once we know $T$ from (3), we form $P(\lambda)$ according to eq. (1) and find an intensity multiplier via

$$
I=\left((P(\lambda))^{T} P(\lambda)\right)^{-1}(P(\lambda))^{T} E(\lambda)
$$

where ${ }^{T}$ is matrix transpose. The associated Planckian is shown dashed in red in Fig. 1(a). From Fig. 1(a) we see, of course, that a single-parameter characterization of a whole spectrum is at best a coarse descriptor.

In terms of perceived colour error, we can make use of CIELAB error [1]. Let us agree to normalize each input spectrum, for example our input fluorescent spectrum $E(\lambda)$, such that $E(\lambda)=100$ at $\lambda=560 \mathrm{~nm}$. Again adopting the 1931 standard observer $X Y Z$ colour-matching 
functions [1] and taking as normalizing illuminant the standard daylight D65, again set equal to 100 at $\lambda=560 \mathrm{~nm}$, we find that using the Planckian from eq. (3) rather than the actual spectrum $E(\lambda)$ results in a CIELAB error [1] $\Delta E=25.94$, quite a large error.

In the next sections, we discuss how to incorporate the requirement that a correlate Planckian spectrum be not just close in colour but also close in terms of the squared error over the entire visible part of the spectrum. We shall see that in fact including spectral error can also improve colour error, compared to the chromaticity-based methods.

To begin that discussion, suppose we start by considering, as a driving objective in an optimization for finding the best $T$, the idea of simply minimizing CIELAB error, ignoring error over the whole spectrum for now.

\section{Best CIELAB Error}

In some circumstances we may wish to generate a Planckian spectral approximation that produces a best fully 3-D CIELAB error, not just a closest chromaticity. Here we use the standard approximately perceptually uniform colour space CIELAB, although we could have used the similar space CIELUV — in general both formulae make similar predictions [1] and we found that to be the case here as well. Moreover, the imaging community is more familiar with the former and as well some difficulties have been identified for the latter [20,21].

Let $I$ be the magnitude of the signal, to be multiplied by a Planckian $\hat{P}(\lambda)$ which we normalize by defining $\hat{P}(\lambda)$ as achieving a maximum of 1.0. Then we aim at minimizing the following objective function:

$$
\min _{T, I}\left\|L A B_{P}-L A B_{E}\right\|^{2}
$$

We can derive a closest Planckian to the actual spectrum $E(\lambda)$ as follows:

$$
\text { Let } P(T, \lambda)=I \cdot \hat{P}(T, \lambda) \text {. }
$$

For input spectrum $E(\lambda)$ and for its approximation $P(T, \lambda)$, the tristimulus values are

$$
X Y Z_{E}=Q^{T} E(\lambda), \quad X Y Z_{P}=Q^{T} P(T, \lambda)
$$

where $Q$ is the set of XYZ colour-matching functions, yielding

$$
L A B_{E}=C I E L A B(E(\lambda), D 65), \quad L A B_{P}=C I E L A B(P(T, \lambda), D 65)
$$

where we convert XYZ to LAB in the standard way [1], normalizing by standard illuminant D65. Now we can write an optimization in eq. (5) above.

The optimization (5) forms a convergent nonlinear program that can be solved using the simplex search method of Lagarias, Reeds, Wright, and Wright [22]. 
Now we find quite a different $T$ for our example spectrum, viz. $T=7617 \mathrm{~K}$, and a CIELAB error $\Delta E=20.91$, still large, but substantially better than simply using the temperature based on chromaticity from Hernández-Andrés, Lee, and Romero [18].

Note that the intensity $I$ found by the optimization eq. (5), 140.74, is very different from the value 108.39 that would be found using the least-squares based multiplier (4) for this $T$, demonstrating that an optimization such as eq. (5) is more than simply a non-chromaticity based method for obtaining a CCT, but is in fact a more novel approach that requires an intensity/temperature pair, $I, T$, to fully describe a spectrum. In a sense, this approach forces $T, I$ into the role of parameters in a particular (nonlinear) curve-fitting, rather than $T$ being implicitly linked to a statistical mechanics based quantum temperature.

This best-CIELAB closest Planckian is shown as dot-dashed magenta in Fig. 1(a). Optimizing on CIELAB error is quite a different approach from [18], and leads to different results. Nevertheless, neither for this method nor for the method in [18] does the Planckian approximation takes into account the large spikes in the illuminant spectrum. This is because only the response of the signal filtered by the $\mathrm{XYZ}$ colour-matching functions produces the $\mathrm{XYZ}$ and $\mathrm{LAB}$ values found. That is, the value of $T$ returned in either method can only take into account that part of the spectrum falling within the Human Visual Subspace [23],

$$
E_{H V S}(\lambda)=\boldsymbol{Q}\left(\boldsymbol{Q}^{T} \boldsymbol{Q}\right)^{-1} \boldsymbol{Q}^{T} E(\lambda)
$$

and this in fact removes the spikes. $E(\lambda)$ and $E_{H V S}(\lambda)$ are plotted in Fig. 1(b). To take these spikes into account, we would have to utilize a different objective function, one that also considers RMS error for the whole spectrum.

For the best-CIELAB optimization approach (5), the temperature found is quite different than for (3), but in fact the colour error is better. In the next section we ask what would be the situation if indeed we were to ask for the least RMS error in terms of the whole spectrum, not simply colour. It should be noted that for the red curve in Fig. 1(a) deriving from the chromaticitybased equation (3) the relative RMS error from the input spectrum $E(\lambda)$ over the entire visible spectrum,

$$
R M S=100 \cdot\left\{\sum[E(\lambda)-I \cdot P(T, \lambda)]^{2}\right\}^{1 / 2} /\left\{\sum[E(\lambda)]^{2}\right\}^{1 / 2}
$$

is $57.70 \%$. For the magenta curve deriving from the best-CIELAB optimization eq. (5) this spectrum RMS error is larger (worse), 61.07\%, which is not surprising since for that curve we emphasize colour error and do not worry about spectral error. These values for $T$ and colourand spectral-errors are collected in Table 1 (where note that so far we have explicated only the first two rows, but for simplicity also collect here the results following below). 


\section{Best Least Squares Blackbody}

Suppose now we emphasize making the whole-spectrum error as small as possible, without worrying about colour error. The classic approach to this problem [2] involves generating a best approximation $P(\lambda)$ to a given spectrum $E(\lambda)$ by setting the temperature $T$ in $P(\lambda)$ such that the ratio of $E(\lambda)$ to $P(\lambda)$ is as close to 1.0 as possible, with the resulting $T$ termed the Distribution Temperature DT. To facilitate this closeness, Robertson [2] includes a scalar intensity $I$ in the optimization, with objective function as follows:

$$
\min _{T, I} \int\left[1-\frac{E(\lambda)}{I \cdot P(\lambda)}\right]^{2} d \lambda
$$

Let us denote the DT thus generated as $T_{D}$.

But now suppose we apply this idea to the spectrum $E(\lambda)$ in Fig. 1(a). In Fig. 2 we repeat the plot of $E(\lambda)$ and the SPD from the closest Planckian derived from the CCT using the method of [18] (red dashed). Recall that for this method the CCT is $T=9354 \mathrm{~K}$, and the CIELAB $\Delta E$ error is 25.94 . Also, we note that the RMS error over the whole spectrum is $57.70 \%$. Now we also plot (magenta dot-dashed) the result using the Distribution Temperature: the optimization yields $T_{D}=5378.1 \mathrm{~K}$. The DT result is unduly influenced by the spikes in the spectrum and also by near-zero values, and now not only is the $\Delta E$ error worse but so is the RMS error: $\Delta E=32.68$ and RMS error $=75.70 \%$, as shown in Table 1 . So, while ostensibly the idea of using the DT is in fact to minimize RMS error we find that, in this case, the error is worse. This problem persists for a large set of measured illuminants as well (as shown below in $\S 6$ ).

So instead, suppose we wished to minimize the spectral difference without using a fraction, directly:

$$
\min _{T, I}\|E(\lambda)-I \cdot P(T, \lambda)\|^{2}
$$

Now we arrive at a different temperature again, $\mathrm{T}=7256 \mathrm{~K}$, and CIELAB error $\Delta E$ of 23.08 units (as shown in Table 1).

Fig. 2 shows the Planckian found using the above minimization (green solid line). The dashed red curve is again for the chromaticity-based method eq. (3) and the magenta dot-dashed curve is for the result of the best-DT based optimization eq. (8). In the case of finding the best RMS value, the CIELAB $\Delta E$ error is again better (less) than that found using eq. (3) but more, of course, than the best CIELAB error derived using a minimization eq. (5) aimed solely at reducing this error.

We find that in this case of finding the least full-spectrum error, the RMS relative wholespectrum error is now $55.72 \%$, the lowest spectrum-error val ue found - and this is not surprising because our objective function took only this error into account.

Notwithstanding the fact that the RMS optimization is very fast, in Appendix 1 we show that in this special case of minimizing whole-spectrum RMS error, eq. (9), we can in fact solve for 
both $T$ and I analytically, and thus need not even employ optimization software at all.

In the next section, we consider how to trade off the two objectives: minimize RMS wholespectrum error on the one hand, and minimize CIELAB error on the other hand. In this case, we do not use an analytic method but instead rely upon a numerical optimization, with the tradeoff specifically encoded in a parameter $\mu$. Moreover, we show how to choose the best-tradeoff $\mu$.

\section{CIELAB versus RMS Spectral Error}

\section{A. Combining Errors}

We would like to combine the two optimization objective functions (5) and (9), so as to determine what the tradeoff is between these two goals. A straightforward approach is to write both objective values as a relative percentage, and then combine the two using a scalar multiplier which we denote here by $\mu$ :

$$
\begin{aligned}
& \min _{T, I}\left\{\sum_{\lambda}[E(\lambda)-I \cdot P(T, \lambda)]^{2}\right\} /\left\{\sum_{\lambda}[E(\lambda)]^{2}\right\} \\
& +\mu\left\{\sum\left[L A B_{E}-L A B_{P}\right]^{2}\right\} /\left\{\sum\left[L A B_{E}\right]^{2}\right\}
\end{aligned}
$$

where the summation for $L A B$ is over the 3-vector components.

Again using our exemplar illuminant spectrum $E(\lambda)$ in Fig. 1, the blue curve in Fig. 3 shows the (absolute) CIELAB $\triangle \mathrm{E}$ error versus (relative) RMS percentage spectral error for an optimization solution for eq. (10) as we let $\mu$ take values from 0 to 100: naturally, as $\mu$ increases and we emphasize the CIELAB error over the RMS spectral error, the latter increases while the former decreases. The red ball shows the position of the least spectral error (for $\mu=0$ ). The green ball shows the result of an optimization that does not include spectral error at all, but only considers CIELAB error - in this case we find a CIELAB error of 20.9 units as stated above (the best value found), and spectral RMS error of 61.07\%. The squares in Fig. 3 show errors that correspond to using the chromaticity-based formulas (2) (black square) and (3) (magenta square). In both cases, the spectral error is reasonable (for this input spectrum) but the CIELAB errors are a good deal worse than using an optimization.

Given the wide range of possible solutions indicated in Fig. 3, in the next section we consider which we should select as the best single representative solution (with errors for our proposed best-tradeoff solution shown as a yellow ball in Fig. 3).

\section{B. Best Multiplier Value}

As an aid in finding the value of the scalar multiplier $\mu$ to use in forming a single representative Planckian for our problem, we note that in Fig. 3 the spectral RMS error first decreases rapidly and then levels off. Let us take the point where the maximum change in RMS error occurs as an 
indicator of a single representative Planckian for our problem. Fig. 4 shows this derivative, and we find that the associated position of the $\mu$ value is $\mu=3.0$.

Error values for this best-guess solution are shown as the yellow ball in Fig. 3; for this solution we have a temperature $T=7392 \mathrm{~K}$, CIELAB error $\Delta \mathrm{E}=21.94$ units, and spectral RMS error $56.22 \%$. So in effect we have settled for a middle balance between spectral error and CIELAB error.

It is interesting to see the range of Planckian spectra represented by the solutions whose errors are shown in Fig. 3. Fig. 5 shows these curves, with the green curve at the top showing the bestCIELAB solution and the red curve on the bottom showing the best spectral-RMS solution. Temperature values range from $T=7617 \mathrm{~K}$ for the green curve to $T=7256 \mathrm{~K}$ for the red curve.

Fig. 6 shows how the various derived tristimulus values are distributed in the chromaticity plane: the closest in chromaticity is the solution from eq. (3) (red diamond); the results of the best-CIELAB optimization ( $\S 3$, green triangle), the best-RMS optimization ( $\S 4$, magenta triangle), and representative optimization ( $\S 5 . \mathrm{B}$, blue star) are also shown. The different optimization results are close to each other whereas the chromaticity-based formula is quite different.

\section{C. Results for Illuminant D65}

Having tackled the quite difficult spiky fluorescent spectrum above, we can carry out the same procedures for the smoother spectrum D65. Now the older formula (2) yields the familiar temperature $T=6504$, whereas (3) yields $T=6500$. For the latter, we find a CIELAB error of $\Delta E=4.23$ and a spectral error of $13.88 \%$.

The best-CIELAB optimization achieves a smaller CIELAB error of $\Delta E=3.30$ and a slightly smaller RMS spectral error of $13.45 \%$. In comparison, the best possible, optimized, spectral RMS error is found to be the smaller value $11.44 \%$, with associated CIELAB error of 5.27 units. So we can see that for this example, the optimization makes less of a difference in terms of error than for the spiky spectrum. However, the CIELAB-driven optimization arrives at quite a different CCT - the value calculated is $T=6312 \mathrm{~K}$, an unfamiliar value that nonetheless does a better job of describing a closest Planckian, in part because of the introduction of a new free parameter $I$, the intensity.

Figs. 7(a,b) show the equivalent plots to those above, now applied to the D65 spectrum.

\section{Experiments over 102 Illuminants}

Applying our best-guess approach from $\S 5$.B above, let us consider the set of 102 illuminants provided by Barnard [24]. These are both fluorescent (i.e., indoor) and non-fluorescent (i.e., outdoor) lights, There are 25 fluorescent illuminants and 77 non-fluorescent illuminants. This experiment makes use of the $196410^{\circ}$ standard observer.

Fig. 8(a) shows a histogram of the difference between spectral errors determined by the algo- 
rithm proposed here, for the best-guess single solution identified in $\S 5$.B above, minus the spectral error for Planckians given by the chromaticity-based formula (3) - i.e., the more negative is the difference the more our proposed PRT outperforms method [18]. In Fig. 8 we separate results for fluorescent and non-fluorescent illuminants via respectively red and blue stacked bars. We note that this difference is mostly negative - the errors found by the proposed algorithm are usually smaller than those found using just the chromaticity. Fig. 8(b) shows a similar histogram for differences between CIELAB errors found by Planckians determined here, versus those for eq. (3). Again, the errors are mostly negative. Moreover, the histogram demonstrates that sometimes the improvements are very substantial in CIELAB units.

The mean difference, over the 102 test illuminants is $-2.54 \%$ for spectral error and $\triangle \mathrm{E}=-2.44$ units for CIELAB error. In other words, the algorithm proposed here has a substantial beneficial effect on both types of errors. Also, compared to the Distribution Temperature DT, the mean difference in RMS error is $-7.81 \%$ and mean $\Delta \mathrm{E}$ difference is -4.27 units, showing that the new PRT substantially outperforms the DT as well.

Breaking out these results for fluorescent and non-fluorescent lights, we find that both the method in [18] and the PRT method perform better for non-fluorescent lights. Table 2 shows both mean CIELAB error results and mean RMS errors, using these two algorithms: the PRT approach consistently outperforms the prior method, particularly with respect to CIELAB errors for fluorescent lights.

\section{Conclusion}

In this paper we have suggested the Planckian Regression Temperature (PRT), a new approach to the use of the Planckian family beyond the CCT or the DT, that balances the need for minimum colour error deriving from such a simplified descriptor with the parallel and competing need for accuracy across the spectrum. For a variety of input spectra, we found that using an optimization approach benefited both colour error as well as spectral error, for a substantial majority of illuminants. We found that in none of the cases we tried, including those presented here, did we encounter any problematic representative Planckian curve or $T$ value as output from the PRT algorithm.

We have made available a reference implementation [25] of this method, in (unoptimized) Matlab. On average, for a $2.13 \mathrm{GHz}$ dual-core PC the optimization converges in $0.0357 \mathrm{sec}$.

One advantage of using a program, rather than a rule such as eq. (3), is that different objectives can be used as the need arises by simple changes in the code. For example, a practitioner may wish to utilize a least-median approach (cf. [26]) instead of a least-sum of squares, and this change can be very simply satisfied by a small change in the code. 


\section{Appendix 1: Analytic Solution for Best-RMS Error}

Consider again the optimization problem (9) for the closest Planckian $P(\lambda)$ to a given $E(\lambda)$. To generate an analytic solution it is helpful to utilize Wien's approximation [1] of Planck's Law:

$$
\hat{P}(\lambda, T) \simeq I k_{1} \lambda^{-5} e^{-\frac{k_{2}}{T \lambda}}
$$

Now let us include $k_{1}$ into $I, I \rightarrow I k_{1}$, and find the closest match between measured light $E(\lambda)$ and the model $\hat{P}$. Larger $I$ simply means multiplicatively increasing the light, but we show now that we can in fact use $I$ to obtain $T$.

To minimize spectral error over the visible spectrum our minimization is thus

$$
\min _{I, T}\|\hat{P}-E\|^{2}
$$

for given, test spectrum $E(\lambda)$. Notwithstanding the fact that we shall be using vectors to represent spectra, it is actually simpler to frame the optimization in terms of continuous quantities:

$$
\min _{I, T} \mathcal{I}=\int\left[I \lambda^{-5} e^{-\frac{k_{2}}{T \lambda}}-E(\lambda)\right]^{2} d \lambda
$$

Define $\tau=1 / T$ as a quantity in inverse-temperature units (thus proportional to micro reciprocal degree units). Then taking logarithms, we can write a transformed optimization problem which is linear in $\tau$ :

$$
\mathcal{I}^{\prime}=\int\left[\log I-5 \log \lambda-\frac{k_{2} \tau}{\lambda}-\log E\right]^{2} d \lambda
$$

We can begin by minimizing this objective function with respect to $\log I$ :

$$
0 \equiv \frac{1}{2} \frac{\partial \mathcal{I}^{\prime}}{\partial \log I}=\int\left[\log I-5 \log \lambda-\log E-\frac{k_{2} \tau}{\lambda}\right] d \lambda
$$

so therefore we can solve for $I$ as follows:

$$
\log I \int d \lambda=\int 5 \log \lambda d \lambda+\int \log E d \lambda+\int \frac{k_{2} \tau}{\lambda} d \lambda
$$

Thus writing in terms of averages over wavelength of these quantities, we have

$$
\log I=\overline{5 \log \lambda}+\overline{\log E}+\overline{\left(\frac{k_{2} \tau}{\lambda}\right)}
$$

Now we can solve for $T$ by substituting this $\log I$. From (16), we have

$$
\mathcal{I}^{\prime}=\int\left\{(\overline{5 \log \lambda}-5 \log \lambda)+(\overline{\log E}-\log E)+\tau\left[\overline{\left(\frac{k_{2}}{\lambda}\right)}-\left(\frac{k_{2}}{\lambda}\right)\right]\right\}^{2} d \lambda
$$


so that if we now set the partial derivative with respect to $\tau$ equal to zero, we obtain:

$$
\begin{aligned}
& 0 \equiv \frac{1}{2} \frac{\partial \mathcal{I}^{\prime}}{\partial \tau} \\
&=\int\left\{\left[\overline{\left(\frac{k_{2}}{\lambda}\right)}-\left(\frac{k_{2}}{\lambda}\right)\right](\overline{5 \log \lambda}-5 \log \lambda+\overline{\log E}-\log E)\right\} d \lambda \\
& \quad+\tau\left\{[1-\epsilon] \int\left[\overline{\left(\frac{k_{2}}{\lambda}\right)}-\left(\frac{k_{2}}{\lambda}\right)\right]^{2} d \lambda\right\}
\end{aligned}
$$

where we have added a small constant $\epsilon$ as a Tikhonov regularization term.

Hence we arrive at a solution for $T$ as well.

\section{References}

1. G. Wyszecki and W. Stiles, Color Science: Concepts and Methods, Quantitative Data and Formulas (Wiley, New York, 1982), 2nd ed.

2. A. Robertson, "Computation of correlated color temperature and distribution temperature," J. Opt. Soc. Am. 58, 1528-1535 (1968).

3. N. Tsumura, "Appearance reproduction and multi-spectral imaging," in "AIC Colour 05: 10th Congress of the Int. Colour Assoc.”, (2005), pp. 119-123.

4. E. Angelopoulou, "Objective colour from multispectral imaging," in "ECCV 2000: European Conference on Computer Vision," (2000), pp. 359-374.

5. M. Drew and G. Finlayson, "Multispectral processing without spectra," J. Opt. Soc. Am. A 20, 1181-1193 (2003).

6. D. Brainard and P. Longere, "Simulation of digital camera images from hyperspectral input," in "Vision Models and Applications to Image and Video Processing," , C. van den Branden Lambrecht, ed. (Springer, 2001). http://color.psych.ucsb.edu/simchapter/index.html.

7. P. Vora, J. Farrell, J. Tietz, and D. Brainard, "Image capture: simulation of sensor responses from hyperspectral images," IEEE Trans. Im. Proc. 10, 307-316 (2001).

8. D. Socolinsky and L. Wolff, "Multispectral image visualization through first-order fusion," IEEE Trans. Im. Proc. 11, 923-931 (2002).

9. M. Roula, J. Diamond, A. Bouridane, P. Miller, and A. Amira, "A multispectral computer vision system for automatic grading of prostatic neoplasia," in "Int. Symp. Biomedical Imaging," (2002), pp. 193-196.

10. Q. Wu, L. Zeng, H. Ke, W. Xie, H. Zheng, and Y. Zhang, "Analysis of blood and bone marrow smears using multispectral imaging analysis techniques," in "Medical Imaging 2005: Image Processing," (2005), pp. 1872-1882. 
11. T. Jääskeläinen and J. Parkkinen. University of Eastern Finland Color Group, http://www.multispectral.org/.

12. J. Kainerstorfer, M. Ehler, F. Amyot, M. Hassan, S. Demos, V. Chernomordik, C. Hitzenberger, A. Gandjbakhche, and J. Riley, "Principal component model of multispectral data for near real-time skin chromophore mapping," J. Biomed. Opt. 15, 046007-1-9 (2010).

13. S. Bergner, M. Drew, and T. Möller, "A tool to create illuminant and reflectance spectra for light-driven graphics and visualization," ACM Trans. on Graphics 28, 5:1-5:11 (2009).

14. S. Bergner, T. Möller, M. Drew, and G. Finlayson, "Interactive spectral volume rendering," in "IEEE Visualization," (IEEE, 2002), pp. 101-108. Selected for proceedings cover.

15. CIE, "International Lighting Vocabulary, 4th ed." CIE-17.4-1987 (Joint publication CIE/IEC) (1987).

16. D. Judd, "Sensibility to color-temperature change as a function of temperature," J. Opt. Soc. Amer. 23, 7-14 (1933).

17. C. McCamy, "Correlated color temperature as an explicit function of chromaticity coordinates," Color Res. Applic. 17, 142-144 (1992). (plus erratum, vol. 18, no. 2, pp.150, 1993).

18. J. Hernández-Andrés, R. Lee, and J. Romero, “Calculating correlated color temperatures across the entire gamut of daylight and skylight chromaticities," Applied Optics 38, 57035709 (1999).

19. Y. Ohno and M. Jergens, "Results of the intercomparison of correlated color temperature calculation," Council for Optical Radiation Measurements (1999). CORM Subcommittee CR3 Photometry.

20. D. Alman, R. Berns, G. Snyder, and W. Larson, "Performance testing of color difference metrics using a color-tolerance dataset," Color Research and Applic. 21, 174-188 (1989).

21. M. D. Fairchild, Color Appearance Models (Addison-Wesley, 1998).

22. J. Lagarias, J. Reeds, M. Wright, and P. Wright, "Convergence properties of the NelderMead simplex method in low dimensions," SIAM J. Optimization 9, 112-147 (1998). (plus erratum, vol. 18, no. 2, pp.150, 2007).

23. P. Vora and H. Trussell, "Measure of goodness of a set of color-scanning filters," J. Opt. Soc. Am. A 10, 1499-1508 (1993).

24. K. Barnard. http://www.cs.sfu.ca/ colour/data/.

25. M. S. Drew and H. R. Vaezi Jose. http://www.cs.sfu.ca/ mark/ftp/PRT/.

26. P. J. Rousseeuw and A. M. Leroy, Robust Regression and Outlier Detection (Wiley, 1987). 


\section{Figure Captions}

Fig. 1

(a): Input spectrum (solid blue), closest Planckian according to $T$ from eq. (3) (dashed red) [T $=9354 \mathrm{~K}]$, and closest Planckian according to the best-CIELAB optimization eq. (5) (dotdashed magenta) [T=7617K]; (b): The part of input spectrum in the HVS (dashed red).

Fig. 2

Input spectrum (solid blue), closest Planckian according to $T$ from eq. (3) (dashed red) $[\mathrm{T}=9354 \mathrm{~K}]$; closest Planckian according to a Distribution Temperatire optimization eq.(8) (dot-dashed magenta) $[\mathrm{T}=5378.1 \mathrm{~K}]$; and best-RMS error optimization eq. (9) (solid green) $[T=7255.6 \mathrm{~K}]$.

Fig. 3

The blue curve is the result of the optimization (10) for values of $\mu$ from 0 (emphasizing minimizing RMS spectral error) to 100 (emphasizing minimizing CIELAB error). The red ball shows the result for $\mu=0$; the green ball shows the result for an optimization for CIELAB error alone. The yellow ball shows a single representative solution ( $\S 5$.B). The black square shows error values using the chromaticity-based formula eq. (2) and the magenta square shows values for eq. (3).

Fig. 4

Derivative value of spectral-RMS error over all multiplier values $\mu$ : the maximum derivative occurs at $\mu=3$ (red dashed line).

Fig. 5

Input spectrum (blue curve) and Planckian optimization solutions over all values of multiplier $\mu$. Green curve at top: best-CIELAB solution $[T=7617 \mathrm{~K}]$; Red curve at bottom: best spectral-error solution $[T=7256 \mathrm{~K}]$.

Fig. 6

(a): $\{x, y\}$ coordinates for input spectrum - black square; closest to Planckian locus (dashed black curve) is solution of eq. (3) - red diamond; best CIELAB optimization - green downward triangle; best representative optimization - blue star. (b): Detail view.

Fig. 7

(a): CIELAB versus RMS spectral errors, for illuminant D65. (b): Range of Planckian spectrum solutions, from best-CIELAB at top (green) to best-spectral at bottom (red). 
Fig. 8

(a): Histogram of amounts by which spectral RMS errors for 102 illuminants are less than those using eq. (3). Red shows errors for fluorescent and blue shows errors for non-fluorescent illuminants, in both histograms. The more negative is the amount shown the better has the PRT performed compared to the CCT. (b): Histogram of amounts by which CIELAB errors are smaller using proposed algorithm. 
Figures 


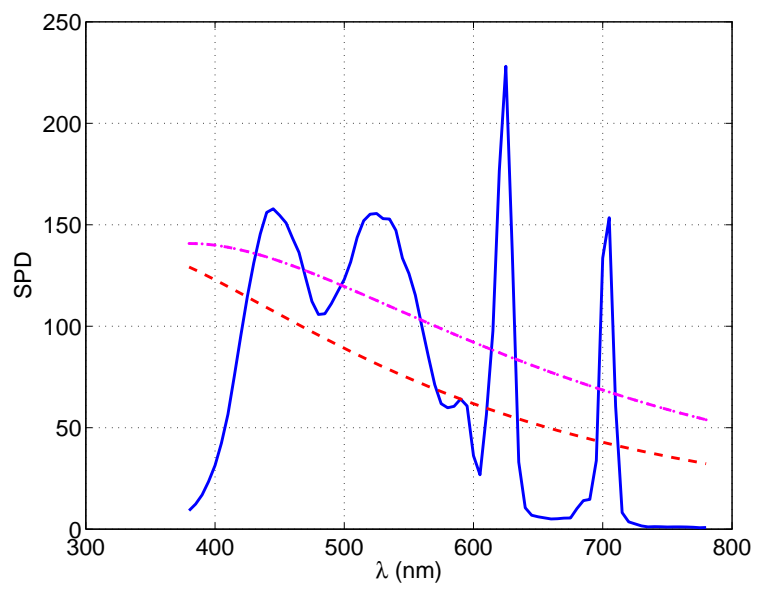

(a)

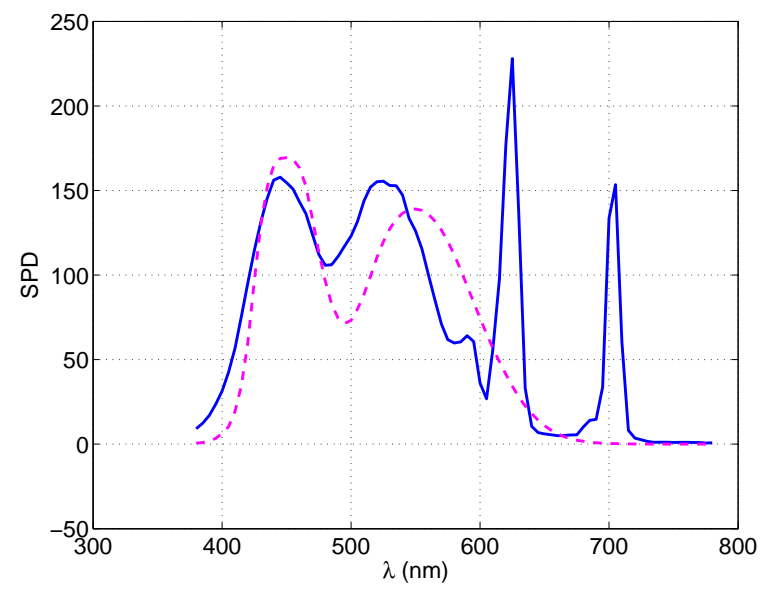

(b)

Fig. 1. (a): Input spectrum (solid blue), closest Planckian according to $T$ from eq. (3) (dashed red) $[\mathrm{T}=9354 \mathrm{~K}]$, and closest Planckian according to the bestCIELAB optimization eq. (5) (dot-dashed magenta) [T=7617K]; (b): The part of input spectrum in the HVS (dashed red). 


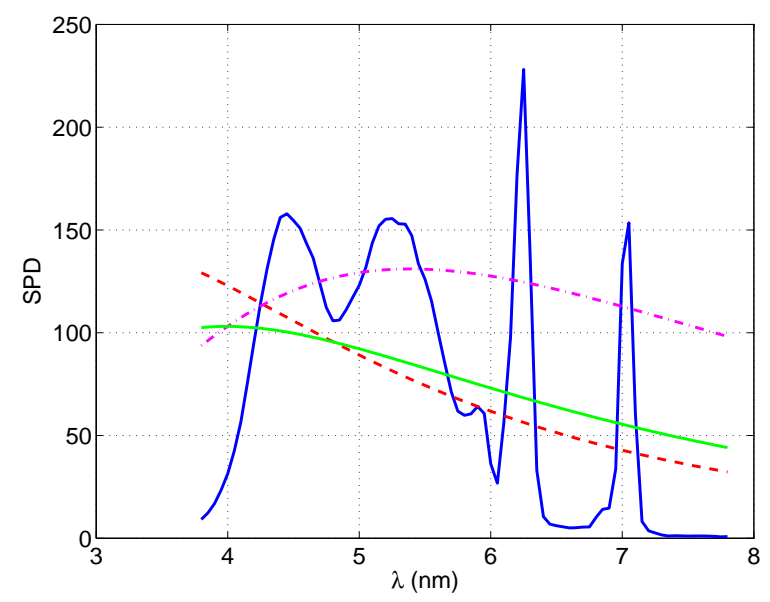

Fig. 2. Input spectrum (solid blue), closest Planckian according to $T$ from eq. (3) (dashed red) $[\mathrm{T}=9354 \mathrm{~K}]$; closest Planckian according to a Distribution Temperatire optimization eq.(8) (dot-dashed magenta) [T=5378.1K]; and best-RMS error optimization eq. (9) (solid green) $[T=7255.6 \mathrm{~K}]$.

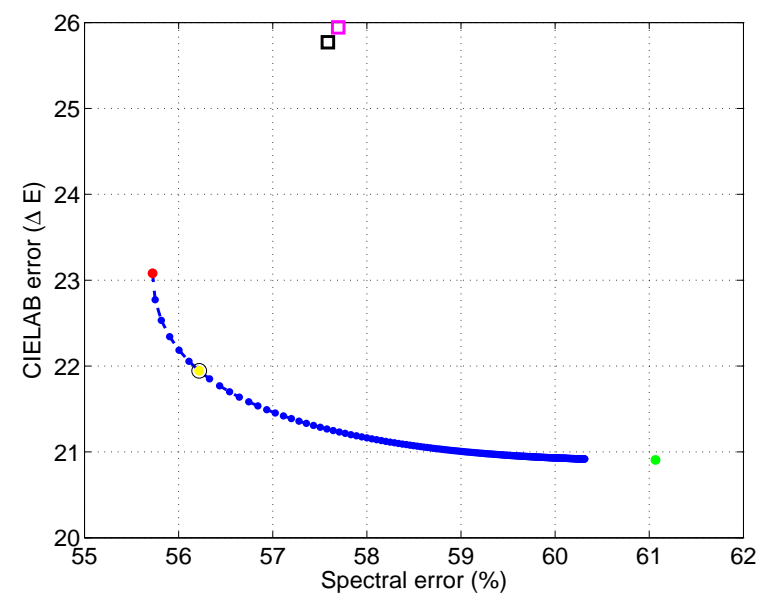

Fig. 3. The blue curve is the result of the optimization (10) for values of $\mu$ from 0 (emphasizing minimizing RMS spectral error) to 100 (emphasizing minimizing CIELAB error). The red ball shows the result for $\mu=0$; the green ball shows the result for an optimization for CIELAB error alone. The yellow ball shows a single representative solution ( $55 . \mathrm{B})$. The black square shows error values using the chromaticity-based formula eq. (2) and the magenta square shows values for eq. (3). 


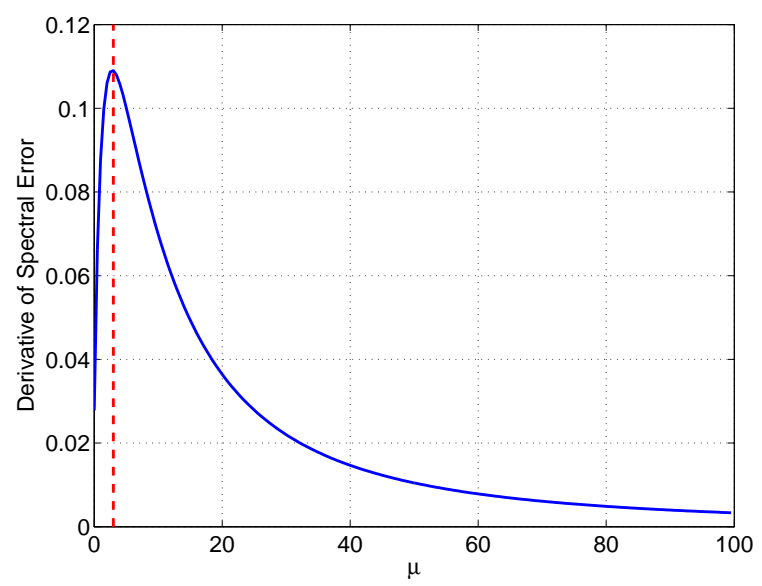

Fig. 4. Derivative value of spectral-RMS error over all multiplier values $\mu$ : the maximum derivative occurs at $\mu=3$ (red dashed line).

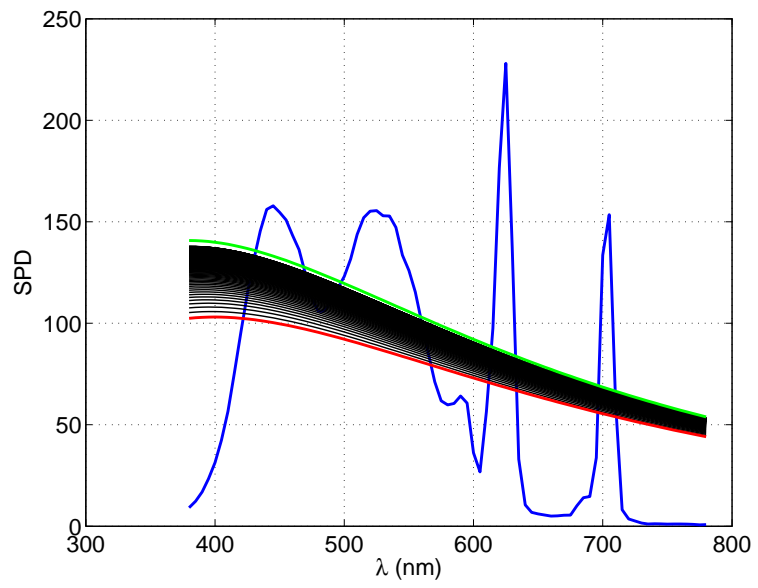

Fig. 5. Input spectrum (blue curve) and Planckian optimization solutions over all values of multiplier $\mu$. Green curve at top: best-CIELAB solution [T=7617K]; Red curve at bottom: best spectral-error solution [ $T=7256 \mathrm{~K}]$. 


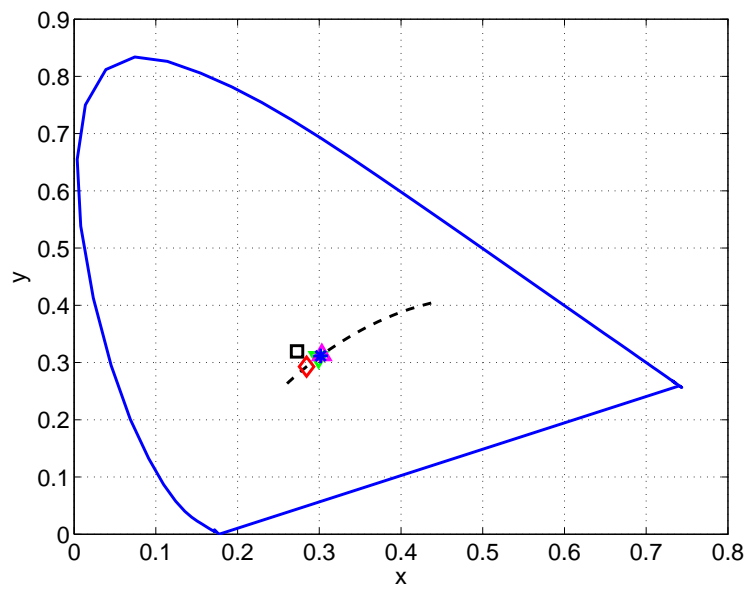

(a)

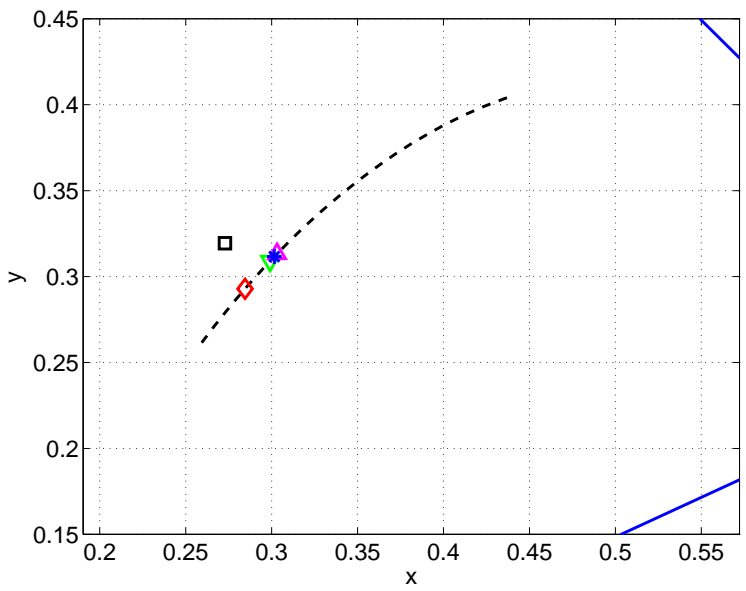

(b)

Fig. 6. (a): $\{x, y\}$ coordinates for input spectrum - black square; closest to Planckian locus (dashed black curve) is solution of eq. (3) - red diamond; best CIELAB optimization - green downward triangle; best representative optimization - blue star. (b): Detail view.

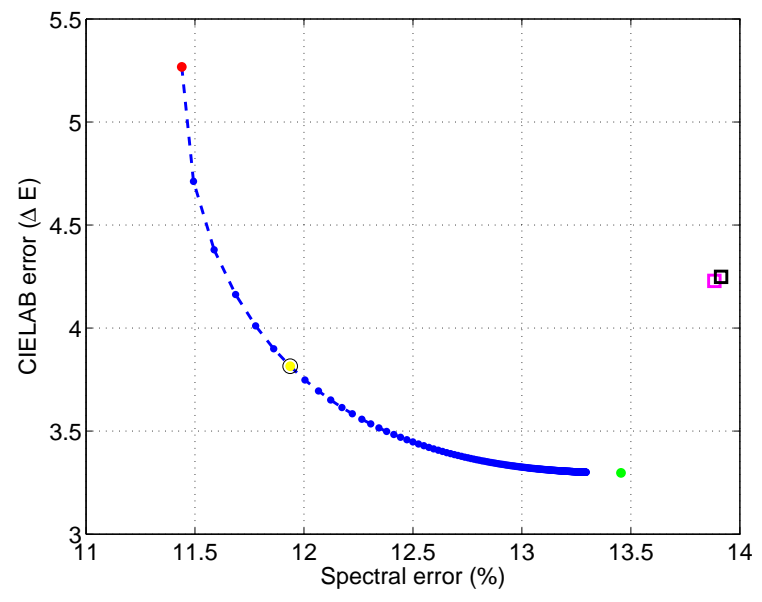

(a)

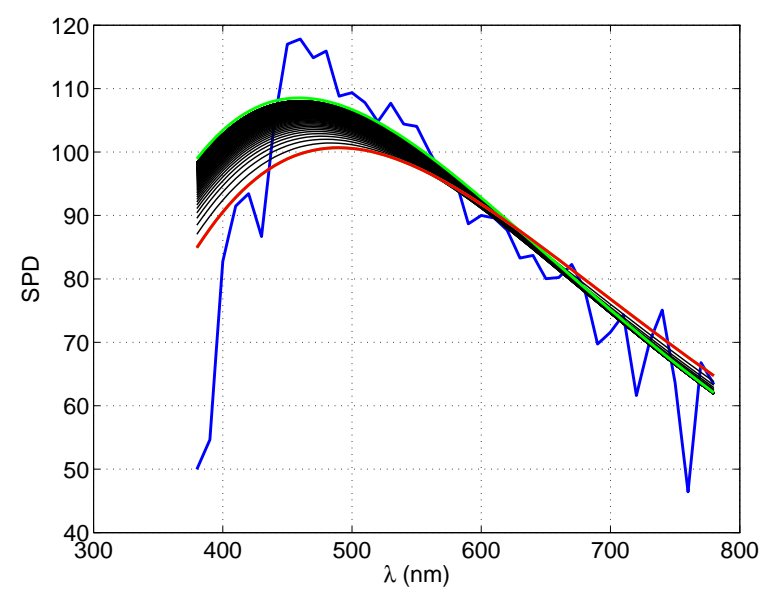

(b)

Fig. 7. (a): CIELAB versus RMS spectral errors, for illuminant D65. (b): Range of Planckian spectrum solutions, from best-CIELAB at top (green) to best-spectral at bottom (red). 


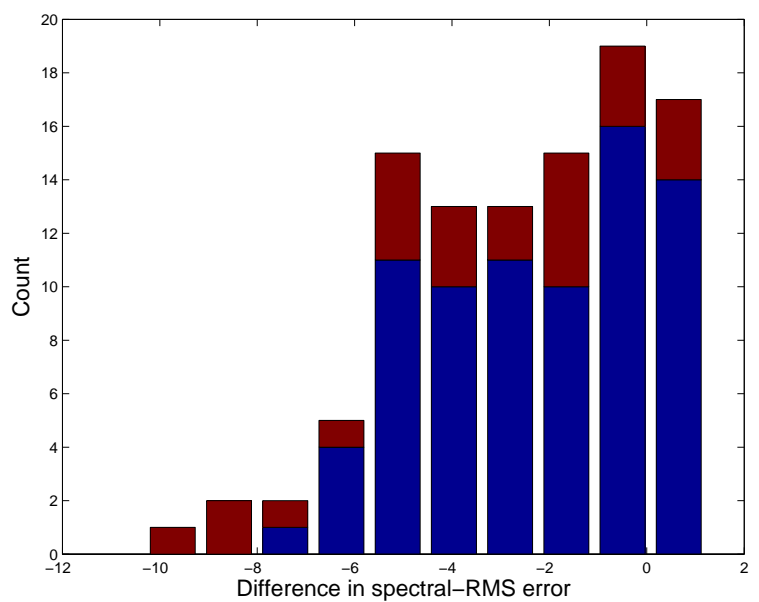

(a)

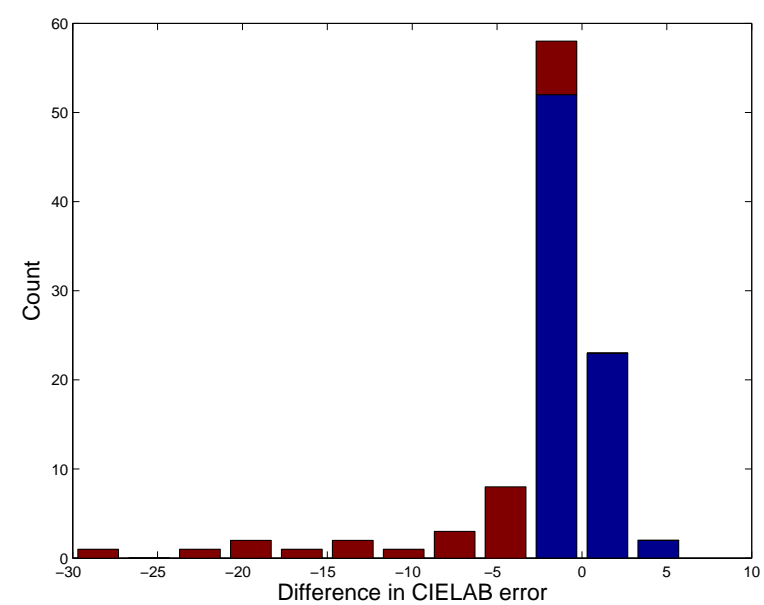

(b)

Fig. 8. (a): Histogram of amounts by which spectral RMS errors for 102 illuminants are less than those using eq. (3). Red shows errors for fluorescent and blue shows errors for non-fluorescent illuminants, in both histograms. The more negative is the amount shown the better has the PRT performed compared to the CCT. (b): Histogram of amounts by which CIELAB errors are smaller using proposed algorithm. 


\section{Tables}

\begin{tabular}{|l|r|r|r|r|}
\hline \multicolumn{5}{|c|}{ Fluorescent Spectrum } \\
\hline Method & $T(\mathrm{~K})$ & $I$ & $\Delta E$ & $\% \mathrm{RMS}$ \\
\hline$[18]$ & 9354 & 129.16 & 25.94 & 57.70 \\
Best-LAB & 7617 & 140.74 & 20.91 & 61.07 \\
Best-RMS & 7256 & 103.07 & 23.08 & 55.72 \\
Best-DT & 5378 & 131.02 & 32.68 & 75.70 \\
$\mu=3.0$ & 7392 & 114.29 & 21.94 & 56.22 \\
\hline
\end{tabular}

Table 1. T, I pairs and errors for methods using eq. (3), $\S 3, \S 4$, and $\S 5$.B. 


\begin{tabular}{|l|c|c|c|c|}
\hline & \multicolumn{2}{|c|}{ Method [18] } & \multicolumn{2}{c|}{ PRT method } \\
& $\Delta E$ & \%RMS & $\Delta E$ & \%RMS \\
\hline 102 Illuminants & 14.40 & 35.34 & 11.96 & 32.80 \\
Fluorescent & 34.27 & 70.40 & 25.71 & 66.98 \\
Non-Fluorescent & 7.95 & 23.95 & 7.50 & 21.70 \\
\hline
\end{tabular}

Table 2. Comparison of mean CIELAB errors and mean RMS errors, collectively and separately for fluorescent and non-fluorescent lights over 102 test illuminants. Difference between (smaller error) PRT results, minus errors found using the method in [18], are shown as negative numbers in Fig. 8. 